\title{
THE CONTRAST BETWEEN VENOGRAPHIC AND CLINICAL ENDPOINTS IN TRIALS OF THROMBOPROPHYLAXIS IN HIP REPLACEMENT
}

\author{
D. Warwick, M. M. Samama
}

In clinical medicine we should be interested in clinical outcomes. Much of the research on thromboembolism in orthopaedic surgery has been based upon the venogram. ${ }^{1}$ Venography has certain advantages. It is a well-defined technique ${ }^{2}$ which is relatively simple and easy to carry out. It is more sensitive than non-invasive methods such as plethysmography and ultrasound for the diagnosis of asymptomatic thrombosis. ${ }^{3,4}$ It also detects 'silent' thrombosis, which is important because most fatal emboli derive from asymptomatic proximal leg clots. Venography, however, remains a surrogate outcome, used because clinical thromboembolic outcomes, apart from death, are difficult to study. The symptoms and signs of a deep-vein thrombosis (DVT) are non-specific and less than 20\% occur during hospitalisation. Those of chronic venous insufficiency are equally so and there is a long lead-time between operation and the outcome of a swollen ulcerated leg. It is only when we understand the frequency at which symptoms occur that we can make a balanced judgement on cost-effectiveness and of the risk of any particular method of prophylaxis. Surrogate outcomes can be misleading in other aspects of medicine ${ }^{5}$ and there are indeed some drawbacks to venography. The procedure has not been fully validated and we have no evidence that the high incidence of DVT detected by venograms reflects a high rate of symptoms. Although the incidence of DVT as detected by venography can be modified by prophylaxis we do not know how it can predict the modification of the symptomatic event rate.

Prevalence rather than incidence. Since it is invasive, uncomfortable and possibly thrombogenic, repeated venography is impractical. A single venogram can only measure prevalence, the rate at the moment when the test is carried out, rather than incidence, the total rate in the postoperative period. This discrepancy is important. First, if the peak onset of DVT is influenced by prophylaxis, then the venogram will not provide a true comparative rate (Fig. 1). Secondly, it will not detect thrombi which have formed and resolved or embolised before the venogram, nor will it

D. Warwick, MD, FRCS, FRCS Orth, Consultant Orthopaedic Surgeon Southampton University Hospitals NHS Trust, Tremona Road, Shirley, Southampton, SO16 6YD, UK.

M. M. Samama, MD, Emeritus Professor of Haematology

Hôtel Dieu de Paris, 1, Plas du Paris, Notre Dame, 75181 Paris Cedex 04, France.

Correspondence should be sent to Mr D. Warwick.

(C)2000 British Editorial Society of Bone and Joint Surgery $0301-620 X / 00 / 49876 \$ 2.00$

J Bone Joint Surg [Br] 2000;82-B:480-2. detect those destined to occur afterwards. ${ }^{6}$ To reduce the discrepancy between incidence and prevalence, testing should be repeated on several occasions. Ultrasound has the advantage of being non-invasive, safe and repeatable, although it is expensive and time-consuming, but at present, it is not accepted as being sufficiently accurate to replace venography, although the accuracy is likely to be improved by the experience of the technician ${ }^{7}$ and better equipment. ${ }^{8}$ As ultrasound improves in accuracy, it will provide the opportunity for the study of the natural history of thrombosis and the relative effects of different methods of prophylaxis. Repeated ultrasound studies would underestimate the rate of occurrence of symptoms, since significant thrombi would be treated when diagnosed and their potential for clinical expression probably suppressed.

Delayed thrombosis. Anticoagulant prophylaxis may delay the onset of DVT as detected by venography and of pulmonary embolism, although it may not reduce the rate. $^{6,9,10}$ If prophylaxis delays the onset of thromboembolism, venography will attribute an inflated benefit to it (Fig. 1).

Late thrombosis. Typically, the venogram is carried out between 7 and 14 days after operation. This choice of timing is not founded on epidemiological or haematological evidence, but rather on convenience, since this period has been, until recently, the typical duration of hospital stay after hip replacement. Although many risk factors which may initiate thromboembolism occur in the perioperative period when the femoral vein is kinked during surgery, ${ }^{11}$ coagulation is activated and fibrinolysis is suppressed, ${ }^{7}$ thrombi may not appear for a few weeks after surgery. These would not be detected by a venogram on days 7 to 14 . In two recent level-1 studies ${ }^{12,13}$ patients were randomised to receive either low-molecular-weight heparin or a placebo after a normal venogram on days 7 to 10 after hip surgery. Of the placebo patients $20 \%$ to $25 \%$ had evidence of DVT when a further venogram was carried out between days 28 and 35 after surgery, although the number with a symptomatic thromboembolic event was low. ${ }^{14,15}$ This suggests that conclusions based on the traditional studies using a venogram on days 7 to 14 should be regarded with circumspection, and the tendency for very early discharge from hospital makes the carrying out of the investigation at this time increasingly difficult.

Accuracy. There is no absolute method to confirm the accuracy of venography and therefore kappa analysis of interobserver and intraobserver reproducibility is used. The diagnosis of venographic DVT is only modestly reproducible ${ }^{\text {16-18 }}$ and this interobserver variability influences the 


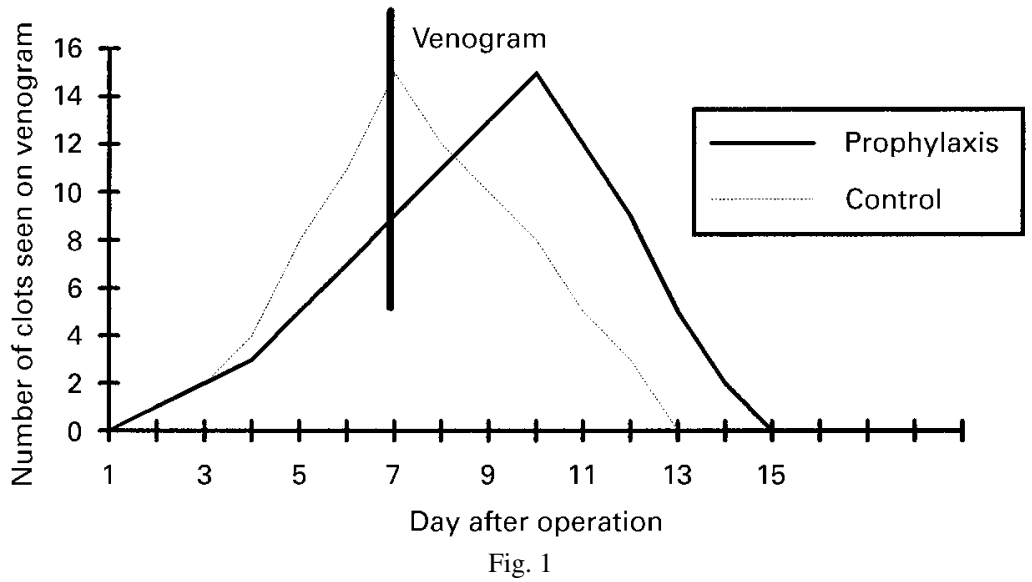

Prevalence or incidence? The number of clots seen on venograms, compared with the day after operation.

reliability of meta-analyses based on venographic data. Generalisability. Each thromboprophylactic study reports a different overall rate of DVT and a different pattern of thrombosis. This may reflect the regimen of prophylaxis, the day chosen for venography, the vulnerability of the study sample to thrombi, the interpretation of venograms and confounding prophylactic methods such as stockings, regional anaesthesia, mobility and surgical technique, which may influence one arm of the study differently from the other. A variability in the incidence of DVT among different centres is well recognised. Thus, there is a need for caution when generalising the results of any one venographic study to another population.

Treatment effects. In venographic studies, patients with a large thrombus, even if asymptomatic, have usually been treated. This alters the natural history and masks the potential for clinical expression of the thrombus as thrombophlebitis or a pulmonary embolism. Patients in such studies may therefore have a better clinical outcome than those to whom the results of the study would be generalised, since the latter would not have had routine venography.

Feasibility. Contrast media and facilities for radiology are expensive, contributing to a large proportion of the cost of clinical trials. Venography is also uncomfortable for the patient. This reduces compliance in trials which require assessment of both legs or repeated investigation. To detect all thrombi bilateral venography is necessary since about $20 \%$ occur in the unoperated leg.

Is a venographic study needed? Before investigating a new regimen of thromboprophylaxis two questions should be asked:

1) Is there a problem with the existing method such as unacceptable rates of symptomatic thromboembolism, cost or complications?

2) Does the new regimen have a realistic prospect of reducing further the rate of symptomatic events without introducing unacceptable complications or expense?

Clinical audit may provide the answer to the first question. This needs a large number of patients to produce usefully narrow confidence intervals and to detect rare, but important complications, particularly death. Patients should be followed for at least three months. ${ }^{19}$ The outcome measures must be clearly defined and objectively recorded. This can be difficult, since most clinical outcomes, apart from death, are varied and non-specific in presentation. Nevertheless, it is only after answering the first question that the second should be considered. This second question should also be answered with clinical outcome measures.

Clinical outcome measures. If audit has suggested the need for better prophylaxis, the most appropriate outcome measure for a subsequent randomised control trial would be the total clinical event rate. Studies on the death rate are limited because they ignore expensive and clinically-relevant events such as symptomatic thromboembolism, chronic venous insufficiency and the side-effects of the drug. A very large sample size is needed. Death is, however, an objective outcome although autopsy rates have greatly decreased and so the cause of death may not be known. If clinically-relevant endpoints are to be used in trials, then their objectivity must be optimised. They can be defined as 'symptomatic events which lead to objective radiological confirmation and subsequent treatment'. A similar definition is already used as the primary outcome in trials of treatment for established thromboembolism. ${ }^{20}$ Wound haematomata could be defined as those confirmed by ultrasound or requiring intervention. The lead-time of two to five years for the development of chronic venous insufficiency and the poor specificity of clinical diagnosis mean that it is unlikely that this could be a primary outcome measure for a clinical trial.

Screening. Because of the difficulties with studies on the rate of symptomatic events, surrogate radiological tests are likely to continue on the assumption that major DVTs correlate with death and chronic venous insufficiency. This is most suitable for phase-II studies in which investigators are trying to compare, objectively, new methods of prophylaxis with reasonably small sample sizes. 
Conclusion. Venography has demonstrated a high rate of DVT after hip replacement, and has allowed objective comparison of different methods of prophylaxis. It may be difficult, however, with existing venographic data to draw clinically relevant conclusions on the most effective form of prophylaxis, its appropriate duration or the most cost- or risk-effective method. Future studies should consider the symptomatic relevance of conclusions based on a radiological surrogate, with a balanced assessment of risk- and cost-benefit. This will require large sample sizes with close definitions of clinical events, or studies with repeated use of a valid surrogate marker.

We wish to thank Dr Jeanne Barre, anaesthetist CHU Maison Blanche, France for her helpful suggestions in the preparation of this manuscript.

\section{References}

1. International Consensus Statement. Prevention of venous thromboembolism. Int Angiology 1997;16:3-38.

2. Lensing AWA, Buller HR, Prandoni P, et al. Contrast venography, the gold standard for the diagnosis of deep vein thrombosis: improvement in observer agreement. Thrombosis and Haemostasis 1992;67:8-12.

3. Warwick DJ, Thornton MJ, Freeman S, et al. Computerized strain gauge plethysmography in the diagnosis of symptomatic and asymptomatic venous thrombosis. Brit J Radiol 1994;67:938-40.

4. Wells PS, Lensing AWA, Davidson BL, Prins MH, Hirsh J. Accuracy of ultrasound for the diagnosis of deep venous thrombosis in asymptomatic patients after orthopaedic surgery: a meta analysis. Ann Intern Med 1995;122:47-53.

5. Fleming TR, DeMets DL. Surrogate end points in clinical trials: are we being misled? Ann Intern Med 1996;125:605-13.

6. Sikorski JM, Hampson WG, Staddon G. The natural history and aetiology of deep vein thrombosis after total hip replacement. $J$ Bone Joint Surg [Br] 1981;63-B:171-7.

7. Garino JP, Lotke PA, Kitziger KJ, Steinberg ME. Deep venous thrombosis after total joint arthroplasty: the role of compression ultrasonography and the importance of the experience of the technician. J Bone Joint Surg [Am] 1996;78-A:1359-65.
8. Kalodiki E, Nicolaides AN, Al-Kutoubi A, Cunningham DA, Crofton M. Duplex scanning in the postoperative surveillance of patients undergoing total hip replacement. J Arthroplasty 1997;12:310-6.

9. Gardlund B. Randomised controlled trial of low-dose heparin for prevention of fatal pulmonary embolism in patients with infectious diseases. Lancet 1996;347:1357-61.

10. Torholm C, Broeng L, Jorgensen PS, et al. Thromboprophylaxis by low-molecular weight heparin in elective hip surgery. J Bone Joint Surg [Br] 1991;73-B:434-8.

11. Warwick DJ, Martin A, Glew D, Bannister GC. Measurement of femoral vein blood flow during total hip replacement: duplex ultrasound with and without the use of a foot-pump. J Bone Joint Surg [Br] 1994;76-B:918-21.

12. Dahl OE, Andreassen G, Aspelin T, et al. Prolonged thromboprophylaxis following hip replacement surgery: results of a doubleblind, prospective randomised placebo-controlled study with dalteparin. Thromb Haemost 1997;77:26-31.

13. Planes A, Vochelle N, Darmon J-Y, et al. Risk of deep vein thrombosis after hospital discharge in patients having undergone total hip replacement: double blind randomised comparison of enoxaparin versus placebo. Lancet 1996;348:224-8.

14. Warwick D, Williams MH, Bannister GC. Death and thromboembolic disease after total hip replacement. J Bone Joint Surg $[\mathrm{Br}]$ 1995;77-B:6-10.

15. Ricotta S, Iorio A, Parise P, Nenci GGF, Agnelli G. Post discharge clinically overt venous thromboembolism in orthopaedic surgery patients with negative venography: an overview analysis. Thromb Haemost 1996;76:887-92.

16. Borris LC, Lassen MR. Venography in deep venous thrombosis: postoperative screening of patients in prophylaxis studies. Haemostasis 1993;Suppl 1,23:80-4.

17. Wille Jorgensen P, Borris L, Jorgensen LN. Phlebography as the gold standard in thromboprophylactic studies. Acta Radiologica 1992;33:24-8.

18. Kalebo P, Ekman S, Lindbratt $\mathbf{S}$, et al. Percentage of inadequate phlebograms and observer agreement in thromboprophylactic multicenter trials using standarized methodology and central assessment. Thromb Haemost 1996;76:893-6.

19. Seagrott V, Tan ST, Goldacre M, Bulstrode C, Nugent I. Elective total hip replacement: incidence, emergency readmission rate, and postoperative mortality. Br Med J 1991;303:1431-5.

20. Levine M, Gent M, Hirsh J, et al. A comparison of low-molecularweight heparin administered primarily at home with unfractionated heparin administered in the hospital for proximal deep-vein thrombosis. N Eng J Med 1996;334:677-81. 